\section{REFERENCES}

1. Abdallah CG, Sanacora G, Duman RS, Krystal JH. Ketamine and rapid-acting antidepressants: a window into a new neurobiology for mood disorder therapeutics. Annu Rev Med. 2015;66:509-23.

2. Joffe ME, Centanni SW, Jaramillo AA, Winder DG, Conn PJ. Metabotropic glutamate receptors in alcohol use disorder: physiology, plasticity, and promising pharmacotherapies. ACS Chem Neurosci. 2018. [Epub ahead of print].

3. Chaki S. mGlu2/3 receptor antagonists as novel antidepressants. Trends Pharmacol Sci. 2017;38:569-80.
4. Bollinger KA, Felts AS, Brassard CJ, Engers JL, Rodriguez AL, Weiner RL, et al. Design and synthesis of mGlu2 NAMs with improved potency and CNS penetration based on a truncated picolinamide core. ACS Med Chem Lett. 2017;8:919-24.

5. Engers $\mathrm{L}$, Bollinger KA, Weiner RL, Rodriguez AL, Long MF, Breiner MM, et al. Design and synthesis of N-Aryl phenoxyethoxy pyridinones as highly selective and CNS penetrant mGlu3 NAMs. ACS Med Chem Lett. 2017;8:925-30.

6. Joffe ME, Santiago Cl, Engers JL, Lindsley CW, Conn PJ. Metabotropic glutamate receptor subtype 3 gates acute stress-induced dysregulation of amygdalo-cortical function. Mol Psychiatry. 2017. [Epub ahead of print].

\title{
Tinkering with THC-to-CBD ratios in Marijuana
}

\author{
Bertha K. Madras (iD) \\ Neuropsychopharmacology (2019) 44:215-216; https://doi.org/10.1038/s41386-018-0217-3
}

The composition of marijuana remains largely unregulated, even though consumption is rising in parallel with rising evidence of harm [1]. The marijuana plant produces over 100 different cannabinoids, including the structurally distinct principals, $\Delta^{9}$-tetrahydrocannabinol (THC) and cannabidiol (CBD). Over two decades, THC concentrations in retail marijuana rose dramatically, while $\mathrm{CBD}$ levels declined, with THC:CBD ratios now 8 times greater than before [2]. U.S. federal guidelines have not been established for THC content or THC:CBD ratios in retail marijuana. The Food and Drug Administration has approved low-dose THC (initial oral dose $0.04 \mathrm{mg} / \mathrm{kg}$ b.i.d.) for treating nausea/vomiting associated with cancer chemotherapy for nonresponders, for treating AIDS-associated anorexia/weight loss, and CBD (initial oral dose $2.5 \mathrm{mg} / \mathrm{kg}$, b.i.d.) to treat rare, severe forms of epilepsy. Smokable marijuana ( 20\% THC; $\sim 0.9 \mathrm{mg} / \mathrm{kg}$ ) delivers THC at $\sim 20$ times the FDA-approved initial dose of oral THC and is now obtainable at THC:CBD ratios varying from 1:1 to 80:1. THC doses and ratios are germane to establishing safety standards, as THC and CBD engender markedly different or even antagonistic molecular, pharmacological and neuropsychiatric effects [3, 4]. Limiting its therapeutic potential, THC in marijuana acutely elicits psychosis, anxiolysis, intoxication, and cognitive impairment. With early initiation and prolonged use, marijuana is addictive and is "likely to increase the risk of developing schizophrenia and other psychoses; the higher the use the greater the risk" [5]. No comparable evidence implicates CBD in engendering euphoria, psychosis, cognitive impairment, anxiety, or addiction. High concentrations of THC and high ratios of THC:CBD in marijuana are associated with more robust euphoria, anxiety, and psychotic symptoms in otherwise normal people. Conversely, CBD mitigates the effects of THC by attenuating anxiety, cognitive deficits or psychosis: (a) in heavy marijuana users consuming a product with high CBD:THC ratios; (b) in marijuana users administered CBD or, (c) in research subjects given $\mathrm{CBD}$ combined with $\mathrm{THC}[4,6,7]$. CBD diminishes the adverse effects of THC by poorly understood processes [3]. One of many possible targets is DCC, which guides formation of frontal cortical dopamine circuits during adolescence and is associated genetically with major psychiatric disorders. In rhesus monkeys treated repeatedly with THC, our pilot data showed upregulation of $d c c$ mRNA in various brain regions, but if administered CBD combined with THC (CBD:THC ratio 3:1), dcc was not elevated [8]. If confirmed with a larger " $n$ ", does THC in marijuana dysregulate $d c c$ expression in human frontal cortex? Does dysregulation alter adolescent brain dopamine circuit formation, thereby contributing to psychosis in susceptible early onset, heavy marijuana users? Our preliminary research is one of many tantalizing leads that warrant comparisons of the pharmacological and pathological consequences of high/low THC doses, high/low THC:CBD ratios and whether CBD can attenuate the effects of a range of THC doses, especially after long-term use. Except for cannabidiol-specific products, most retail marijuana strains contain immoderately high concentrations of THC and scant CBD levels. Accumulating research documents the pitfalls of an unregulated industry producing psychoactive compounds, while operating without a foundation of informed science.

\section{ACKNOWLEDGEMENTS}

The author thanks her indispensable collaborators Dr. Susan George, Dr. Jack Bergman, Dr. Stephen Kohut, Dr. Sarah Withey, Dr. Zhicheng Lin, Dr. Yasmin Hurd, Dr. A. Hasbi, and students Sophia Charles and Andrew Gumbert.

\section{FUNDING}

The research is funded by a grant from the NIH-National Institute on Drug Abuse NIDA: DA042178. The author receives funding for specific speaking engagements focused on the public health challenges of drugs of abuse and solutions.

Competing interests: The author declares no competing interest.

Publisher's note: Springer Nature remains neutral with regard to jurisdictional claims in published maps and institutional affiliations.

\section{REFERENCES}

1. De Aquino JP, Sherif M, Radhakrishnan R, Cahill JD, Ranganathan M, D'Souza DC. The psychiatric consequences of Cannabinoids. Clin Ther. 2018.

2. ElSohly MA, Mehmedic Z, Foster S, Gon C, Chandra S, Church JC. Changes in cannabis potency over the last 2 decades (1995-2014): analysis of current data in the United States. Biol Psychiatry. 2016;79:613-9.

3. Boggs DL, Nguyen JD, Morgenson D, Taffe MA, Ranganathan M. Clinical and preclinical evidence for functional interactions of cannabidiol and $\Delta(9)$-Tetrahydrocannabinol. Neuropsychopharmacology. 2018;43:142-54.

\footnotetext{
${ }^{1}$ McLean Hospital and Department of Psychiatry, Harvard Medical School, Belmont, MA 02478, USA
}

Correspondence: Bertha K. Madras (bertha_madras@hms.harvard.edu, bmadras@partners.org)

Received: 2 August 2018 Accepted: 6 September 2018

Published online: 19 September 2018 
4. Englund A, Freeman TP, Murray RM, McGuire P. Can we make cannabis safer? Lancet Psychiatry. 2017;4:643-8.

5. National Academies of Sciences, Engineering, Medicine. The Health Effects of Cannabis and Cannabinoids: The Current State of Evidence and Recommendations for Research, January 2017. http://nationalacademies.org/hmd/Reports/2017/ health-effects-of-cannabis-and-cannabinoids.aspx; http://nationalacademies.org/ hmd/ /media/Files/Report\%20Files/2017/Cannabis-Health-Effects/Cannabischapter-highlights.pdf.

6. Morgan CJA, Curran HV. Effects of cannabidiol on schizophrenia-like symptoms in people who use cannabis. Br J Psychiatry. 2008;192:306-7.
7. Schubart CD, Sommer IE, van Gastel WA, Goetgebuer RL, Kahn RS, Boks MP (2011). Cannabis with high cannabidiol content is associated with fewer psychotic experiences. Schizophr Res. 2011;130:216-21.

8. Madras BK, Bergman J, Kohut S, George S, Hurd Y, Lin Z. THC of marijuana upregulates dcc mRNA expression in prefrontal cortex but THC combined with Cannabidiol (CBD) does not: relevance to psychiatric symptoms associated with long term marijuana use? American College of Neuropsychopharmacology 56th Annual Meeting. Palm Springs, CA, 3-7 Dec 2016: Poster T234; 2017.

\title{
Sex differences in schizophrenia: estrogen and mitochondria
}

\author{
Vanessa F. Gonçalves ${ }^{1}$, Ari B. Cuperfain ${ }^{1}$ and James L. Kennedy ${ }^{1,2}$ \\ Neuropsychopharmacology (2019) 44:216-217; https://doi.org/10.1038/s41386-018-0228-0
}

Schizophrenia (SCZ) is a neuropsychiatric disorder which displays sex differences in its clinical manifestations, including women presenting with later age at onset, less severe course, and better response to antipsychotics in comparison with men. Estrogen is a steroid hormone which, in addition to its role in females' reproduction and sex characteristics, contributes to neuroprotection within the central nervous system.

In this context, there is compelling evidence that estrogen has a protective effect in females against the development of neuropsychiatric disorders, particularly SCZ. Estrogen is reported to influence several features of SCZ pathophysiology and to promote the observed sex-difference in the clinical outcomes between males and females. The molecular mechanisms by which estrogen affects SCZ are still largely unknown, but there is evidence that estrogen plays a role in synaptic plasticity, neurogenesis, and neurotransmission, as well as in modulating reactive oxygen species (ROS) burden in the brain [1]. Notably, each of these neurobiological mechanisms is disturbed in the brain of SCZ patients.

More recently, it has been reported that estrogen also modulates mitochondrial function [2]. Mitochondria are the main source of energy for most cellular activities. In the brain, mitochondria are also implicated in many neuronal processes reported to be involved in SCZ pathophysiology, and mitochondrial dysfunction is emerging as a risk factor for the disorder [3]. Over 1000 mitochondrial genes are encoded by the nuclear genome, and also, mitochondria contain their own small genome (mtDNA), which is exclusively maternally inherited. Interestingly, studies have revealed a stronger maternal inheritance of SCZ compared to paternal [4].

Mitochondria have sex-specific features, exhibiting distinct effects in males versus females, and these effects have strong links to neuroprotection. Briefly, studies in a variety of animal models have shown that females' mitochondria have increased biogenesis and oxidative capacity, and greater antioxidant defense with (consequently) reduced generation of ROS, thus less release of mitochondrial apoptotic factors [5]. There is evidence that estrogen might be a key player in this mitochondrial sexual dimorphism. Several nuclear-encoded mitochondrial genes show specific DNA sequences that are targets of estrogen, called estrogen response elements (ERE). The strongest evidence is the finding that estrogen receptor beta binds to estrogen EREs in $m t D N A$, directly regulating its gene transcription. Moreover, with respect to direct biochemical connection for the role of estrogen in mitochondrial function, treatment of cells with estrogen protects against electron transport chain inhibitors [2]. However, the actions of estrogen on mitochondrial functioning that may underlie this hormone's sex-specific effects on brain development and behavior in schizophrenia remain uninvestigated, and are an important target of research in animal models for the disease.

Overall, it appears that estrogen plays a role in SCZ pathophysiology and in protecting females against a more severe course of the disease, probably (but not exclusively) through its regulation of the mitochondrial system. A better understanding of the influence of estrogen on mitochondrial function in the context of SCZ will reveal new insights regarding the pathophysiology of the disease in males versus females. This new perspective will generate novel targets for drug discovery with potential to improve many of the SCZ clinical outcomes, as well as developing future preventive and therapeutic strategies.

\section{ACKNOWLEDGMENTS}

We would like to acknowledge the Judy and Larry Tanenbaum Family Foundation for their support to this study.

\section{FUNDING}

J.L.K. has been funded by Genome Canada, The Ontario Ministry of Research and Innovation, CIHR, a BBRF/NARSAD Distinguished Investigator Award, and the Judy and Larry Tanenbaum Foundation. J.L.K. has also received speaker honoraria and expenses from Eli Lilly, Novartis, and Shire, and consultant honoraria and expenses from Roche, and is a nonpaid member of the SAB of Assurex Health Inc.Competing interests: The authors declare no competing interest.

Publisher's note: Springer Nature remains neutral with regard to jurisdictional claims in published maps and institutional affiliations. 